\title{
Estudio en célula Hull de un baño electrolítico de NiW y evaluación de sus propiedades ${ }^{(*)}$
}

\author{
I. García-Urrutia*, J.A. Díez*, C. Müller** y P. Calvillo*
}

\begin{abstract}
Resumen
El interés en los recubrimientos de NiW ha aumentado en los últimos años debido a sus buenas propiedades como dureza, resistencia al desgaste y resistencia a la corrosión, situándolos como una de las alternativas a los actuales recubrimientos de cromo duro. En este trabajo se ha estudiado, mediante el empleo de célula Hull, la influencia de la concentración de metales, la temperatura, el pH y la densidad de corriente sobre la composición de la aleación y su espesor. Asimismo se han estudiado las principales propiedades de los depósitos de NiW como morfología, dureza, y resistencia a la abrasión y a la corrosión.
\end{abstract}

Palabras clave Electrodeposición; Aleación NiW; Célula Hull; Cromo duro; Propiedades NiW.

\section{A Hull cell study of a NiW electrolyte and evaluation of its properties}

\begin{abstract}
Interest in $\mathrm{NiW}$ coatings has grown in recent years due to its favourable properties such as hardness, and resistance to both wear and corrosion, making it one of the actual alternatives to hard chromium coatings. In this work, we have undertaken a Hull cell study, investigating the influence of the metal concentration, temperature, $\mathrm{pH}$ and current density on the composition of the alloy formed and its thickness. We have also studied the most important properties of the $\mathrm{NiW}$ deposits, including morphology, hardness, and resistance to abrasion and corrosion.
\end{abstract}

Keywords

Electrodeposition; NiW alloy; Hull cell; hard chromium; NiW properties.

\section{INTRODUCCIÓN}

El interés en la electrodeposición de aleaciones de wolframio y, en concreto, la de $\mathrm{NiW}$, ha crecido notablemente en los últimos años debido a las excelentes propiedades mecánicas y tribológicas del recubrimiento, que lo hacen apropiado para numerosas aplicaciones industriales. Entre las principales características de los recubrimientos de NiW cabe destacar su alta dureza, su estabilidad térmica y su resistencia a la corrosión y la abrasión, lo que los sitúan como una de las alternativas a los actuales recubrimientos de cromo duro ${ }^{[1]}$.

La electrodeposición de NiW se realiza, según la clasificación realizada por Brenner ${ }^{[2]}$, a través de un mecanismo de codeposición inducida, ya que el wolframio no es capaz de depositarse por sí solo a partir de una disolución acuosa. La presencia de iones níquel en la disolución induce la codeposición conjunta de ambos metales, para formar la aleación de Ni-W. Son diversos los mecanismos de deposición de NiW propuestos hasta la fecha para explicar este comportamiento ${ }^{[3-6]}$; sin embargo, todavía no quedan completamente justificados los mecanismos propuestos. La dificultad radica en la gran cantidad de iones complejos que pueden coexistir en la disolución y que pueden servir de precursores de la deposición de la aleación, especies que varían en función de la composición y de los parámetros de trabajo influyendo, en consecuencia, sobre la composición de la aleación y su rendimiento. En un estudio sistemático del proceso (probablemente el más completo), Younes y Gileadi ${ }^{[3]}$, trabajando con baños base citrato en un rango de $\mathrm{pH} 7-10$ y a una temperatura de $25^{\circ} \mathrm{C}$, han determinado las especies presentes en disolución. En estas condiciones, han determinado que la especie predominante es el ión citrato con 3 cargas negativas $\left(\mathrm{Cit}^{3-}\right)$, formando los complejos mayoritarios

\footnotetext{
(•) Trabajo recibido el día 10 de diciembre de 2007 y aceptado en su forma final el d-ia 13 de noviembre de 2008.

* Departamento de Tratamientos Superficiales, CIDETEC, P'Miramón 196, 20009 San Sebastián, España. igarcia@cidetec.es

** Electrodep. Dpt. Química Física, Universidad de Barcelona, Martí i Franquès 1, 08028 Barcelona, España.
} 
$\left[\mathrm{Ni}(\mathrm{Cit})_{2}\right]^{4-}$ y $\left[\left(\mathrm{WO}_{4}\right)(\mathrm{Cit})(\mathrm{H})_{\mathrm{x}}\right]^{\mathrm{x}-5}$ donde $\mathrm{x}$ puede variar entre 1 y 3 . Sin embargo, los autores postulan que los metales no se depositan a partir de sus respectivos complejos sino que existe un precursor mixto de la codeposición de la aleación $\mathrm{NiW}$, del que no se tiene evidencia experimental, que podría ser un compuesto ternario del tipo $\left[(\mathrm{Ni})\left(\mathrm{HWO}_{4}\right)(\mathrm{Cit})\right]^{2-}$.

Partiendo de los resultados obtenidos por estos autores, en este trabajo se ha realizado un estudio sistemático para optimizar las condiciones de trabajo que permitan obtener depósitos de aleación $\mathrm{NiW}$ con altos porcentajes de wolframio en el depósito (para conseguir una elevada dureza y resistencia a la corrosión) y con un buen rendimiento del proceso (que permita obtener altos espesores en un tiempo razonable).

El estudio se ha llevado a cabo en condiciones galvanostáticas en célula Hull, estudiando la influencia de los diferentes parámetros experimentales sobre las principales propiedades del depósito, como morfología, composición de la aleación, resistencia a la abrasión, resistencia a la corrosión y dureza. No se ha llevado a cabo ningún estudio adicional sobre el mecanismo del proceso, aunque en estudios previos realizados en el laboratorio de los autores, los análisis del electrolito corroboran la presencia de los mismos complejos de níquel y wolframio.

La Célula Hull es uno de los sistemas prácticos más utilizados para el control de los baños galváni$\cos ^{[4}$ y 5$]$. El método consiste en la observación del comportamiento de una pequeña porción del baño que se desea ensayar cuando es sometida a las mismas condiciones de electrólisis que en la cuba real. Una de sus características más notables es que, realizando un solo ensayo a intensidad de corriente constante, se logra que a lo largo del cátodo se produzca una variación progresiva de la densidad de corriente. De esta manera se obtiene sobre un mismo cátodo o probeta la respuesta a una gradación de densidades de corriente, lo cual permite reducir el número de ensayos necesarios para un correcto control del sistema y, al mismo tiempo, obtener una clara idea de la influencia que en la deposición electrolítica ejercen las diferentes variables del proceso sobre el espesor del depósito y la distribución metálica.

\section{EXPERIMENTAL}

\subsection{Experiencias en célula Hull}

La deposición de la aleación de NiW se llevó a cabo a partir de una disolución acuosa conteniendo entre 0,034 y 0,13 M de sulfato de níquel, entre 0,055 y 0,22 M de wolframato sódico, 0,36 M de citrato amónico y 0,3 $\mathrm{M}$ de un ácido orgánico de cadena corta como compuesto estabilizante. $\mathrm{El} \mathrm{pH}$ de las disoluciones se varió entre 5 y 9 . La temperatura de trabajo fue de entre 25 y $75^{\circ} \mathrm{C}$.

Para la realización del estudio en célula Hull, se utilizó una célula termostatizada de $267 \mathrm{~cm}^{3}$ de la casa KOCOUR, con agitación del electrolito mediante aireación. Los cátodos utilizados fueron de latón de dimensiones 10 x 7,5 cm. El ánodo empleado fue de acero inoxidable AISI 316 con una superficie de $25 \mathrm{~cm}^{2}$. La fuente de corriente utilizada fue un rectificador de corriente modelo BLAUSONIC FA 350, aplicando una intensidad de $2 \mathrm{~A}$. El tiempo de deposición fue de $900 \mathrm{~s}$.

Para el cálculo de las densidades de corriente correspondiente a cada punto de medición se aplicó la fórmula definida por Hull-Mac Intere ${ }^{[6]}$ :

$$
D C=I(5,103-5,238 \log L)
$$

en la que DC se obtiene en $\mathrm{A} / \mathrm{dm}^{2}$, I se expresa en amperios y $\mathrm{L}$ (distancia del punto considerado al extremo de máxima densidad de corriente del cátodo) en $\mathrm{cm}$. Los puntos de medición fueron tomados a las siguientes distancias: 0,5, 2,0, 3,5, 5,0, 6,5, 8,0 y 9,5 cm.

\subsection{Experiencias en celda de dos electrodos}

Para llevar a cabo la evaluación de las propiedades, los depósitos de NiW se generaron a partir de una disolución acuosa que contenía $0,16 \mathrm{M}$ de wolframato sódico, 0,10 M de sulfato de níquel, 0,36 M de citrato amónico y 0,3 $\mathrm{M}$ de compuesto estabilizante. El $\mathrm{pH}$ de la disolución fue de 7,0 y la temperatura de trabajo de $65^{\circ} \mathrm{C}$. Se empleó una celda de 2 electrodos de 101 de capacidad. Como ánodos se utilizaron dos láminas de acero inoxidable AISI 316 y una lámina de acero de bajo contenido en carbono como cátodo. La densidad de corriente utilizada fue de 5 $\mathrm{A} / \mathrm{dm}^{2}$. La electrodeposición se realizó durante $1 \mathrm{~h}$ $30 \mathrm{~min}$. El espesor medio del recubrimiento de NiW fue de $25-30 \mu \mathrm{m}$.

La medición del espesor y el porcentaje de la aleación se llevó a cabo empleando la técnica de fluorescencia de Rayos-X (FISCHERSCOPE, modelo XDL), según la norma UNE-EN-ISO 3497. El porcentaje de error del equipo es de un $2 \%$. Los puntos de medición se indican en el apartado 2.1. Para estudiar la morfología de la aleación se utilizó un microscopio electrónico de barrido (SEM, JEOL model JSM5910$\mathrm{LV})$. La resistencia al desgaste se evaluó mediante el método Taber (TABER ABRASER, modelo 5131), empleando ruedas abrasivas CS-10, $500 \mathrm{~g}$ de carga y 
10.000 ciclos. Para las mediciones de microdureza se utilizó un microdurómetro Shimadzu tipo M con una carga de $0,98 \mathrm{~N}(\mathrm{Hv} 0,1)$. La resistencia a la corrosión se evaluó mediante ensayos de niebla salina neutra según la norma UNE 112-017-92.

\section{RESULTADOSY DISCUSIÓN}

\subsection{Estudio en célula Hull}

\subsubsection{Influencia de la concentración de níquel}

Partiendo de un baño compuesto por 0,16 $\mathrm{M}$ de wolframato sódico, 0,36 M de citrato amónico y 0,3 M de compuesto estabilizante, se varió la concentración de níquel entre 0,034 M y 0,13 M. En las figuras 1 y 2 se muestra la variación del espesor del depósito y de la composición de la aleación con la concentración de níquel a diferentes densidades de corriente.

En la figura 1 se puede apreciar que, al aumentar la concentración de níquel hay un aumento claro del espesor del depósito sobre todo a partir de 0,068 M de

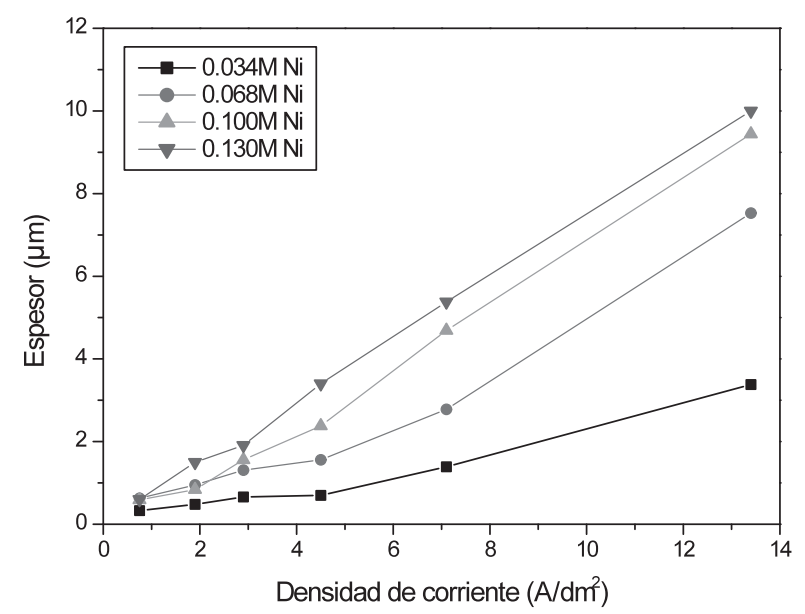

Figura 1. Variación del espesor del depósito de NiW en función de la concentración de níquel, a una intensidad de $2 \mathrm{~A}$ totales. Condiciones de trabajo: 0,034 a $0,13 \mathrm{M}$ de níquel, $0,16 \mathrm{M}$ de wolframio, 0,36 $\mathrm{M}$ de citrato amónico y 0,3 $\mathrm{M}$ de compuesto estabilizante. Temperatura $65^{\circ} \mathrm{C}, \mathrm{pH} 7$.

Figure 1. Variation of the thickness of NiW deposits as a function of nickel concentration, at an intensity of $2 \mathrm{~A}$. Working conditions: 0.034 to $0.13 \mathrm{M}$ of nickel, $0.16 \mathrm{M}$ of wolfram, $0.36 \mathrm{M}$ of ammonium citrate y $0.3 \mathrm{M}$ of estabilizer. Temperature $65^{\circ} \mathrm{C}, \mathrm{pH} 7$.

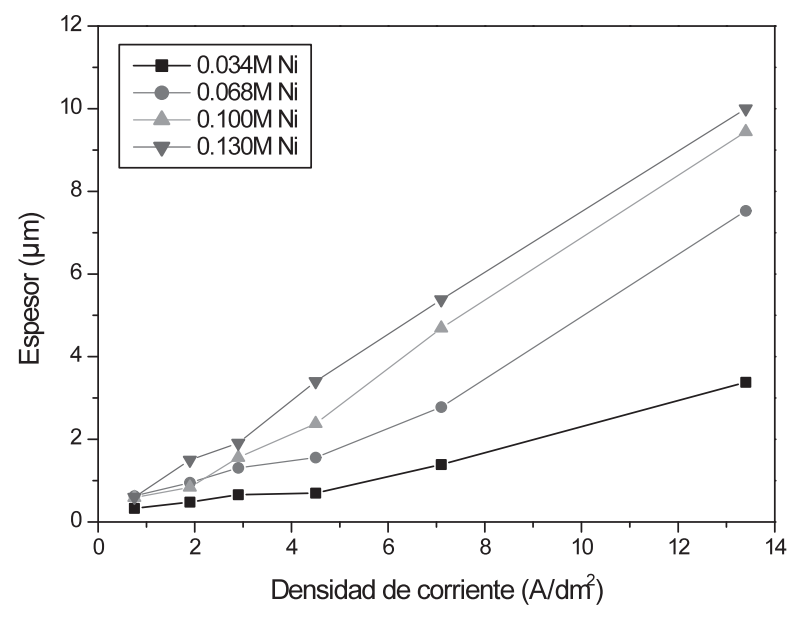

Figura 2. Variación del porcentaje de wolframio (\% en peso) en la aleación de NiW en función de la concentración de níquel, a una intensidad de 2 A totales. Condiciones de trabajo: 0,034 a $0,13 \mathrm{M}$ de níquel, $0,16 \mathrm{M}$ de wolframio, $0,36 \mathrm{M}$ de citrato amónico y $0,3 \mathrm{M}$ de compuesto estabilizante. Temperatura $65^{\circ} \mathrm{C}, \mathrm{pH} 7$.

Figure 2. Variation of wolfram contained in the NiW alloy (\% in weight), as a function of the concentration of nickel, at a total intensity of $2 \mathrm{~A}$. Working conditions: 0.034 to $0.13 \mathrm{M}$ of nickel, $0.16 \mathrm{M}$ of wolfram, $0.36 \mathrm{M}$ of ammonium citrate y $0.3 \mathrm{M}$ of estabilizer. Temperature $65^{\circ} \mathrm{C}, \mathrm{pH} 7$.

níquel en disolución. Con respecto a la densidad de corriente, por debajo de $3 \mathrm{~A} / \mathrm{dm}^{2}$ no hay diferencias apreciables en el espesor. Sin embargo, a partir de 4 $\mathrm{A} / \mathrm{dm}^{2}$ el espesor aumenta de forma más pronunciada. Asimismo, se observa que a bajas concentraciones de níquel $(0,034 \mathrm{M})$ el espesor es bajo en todo el intervalo de densidades de corriente.

En la figura 2 se representa la variación del porcentaje de wolframio de la aleación con respecto a la densidad de corriente y a diferentes concentraciones de níquel en el electrolito. Se puede apreciar que a partir de una densidad de corriente de $4 \mathrm{~A} / \mathrm{dm}^{2}$, el porcentaje de la aleación se mantiene constante, siendo este valor de entre 40 y $50 \%$ wolframio para todas las concentraciones de níquel. Por debajo de $4 \mathrm{~A} / \mathrm{dm}^{2}$ el porcentaje de la aleación fluctúa mucho, habiendo una mayor tendencia del níquel a depositarse.

\subsubsection{Influencia de la concentración de wolframio}

Partiendo de un baño con $0,10 \mathrm{M}$ de sulfato de Níquel, 0,36 M de citrato amónico y 0,3 M de 
compuesto estabilizante, se ha variado la concentración de wolframio entre $0,055 \mathrm{M}$ y 0,22 M. En las figuras 3 y 4 se muestra la variación del espesor del depósito y de la composición de la aleación con la concentración de wolframio a diferentes densidades de corriente.

En la figura 3 se puede apreciar un aumento lineal del espesor al aumentar la densidad de corriente. Además se observa que no hay diferencias apreciables en el espesor al aumentar la concentración de wolframio en el electrolito.

En la figura 4 se puede apreciar que el porcentaje de wolframio en la aleación aumenta al aumentar su concentración en el baño, observándose un salto importante entre $0,055 \mathrm{M}$ y 0,11 $\mathrm{M}$ de wolframio. A partir de esta concentración, el porcentaje de la aleación se mantiene entre un 45 y un 50 \% wolframio en peso. Asimismo, puede verse que hasta una densidad de corriente de $4 \mathrm{~A} / \mathrm{dm}^{2}$, el porcentaje de wolframio va aumentando progresivamente. A partir de esta corriente, el porcentaje de la aleación se mantiene constante en todo el rango de densidades de corriente.

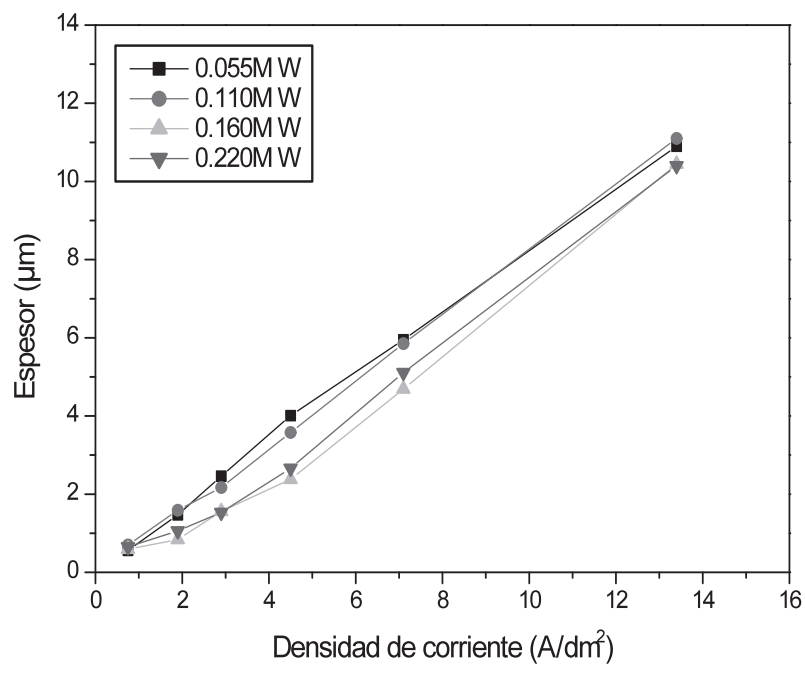

Figura 3. Variación del espesor del depósito de NiW en función de la concentración de wolframio, a una intensidad de 2 A totales. Condiciones de trabajo: $0,10 \mathrm{M}$ de níquel, 0,055 a 0,22 M de wolframio, 0,36 M de citrato amónico y $0,3 \mathrm{M}$ de compuesto estabilizante. Temperatura $65^{\circ} \mathrm{C}, \mathrm{pH} 7$.

Figure 3. Variation of the wolframio thickness of the NiW alloy as a function of the concentration of wolfram, at a total intensity of 2 A. Working conditions: $0.10 \mathrm{M}$ of nickel, 0.055 to $0.22 \mathrm{M}$ of wolfram, $0.36 \mathrm{M}$ of ammonium citrate y $0.3 \mathrm{M}$ of estabilizer. Temperature $65^{\circ} \mathrm{C}, \mathrm{pH} 7$.

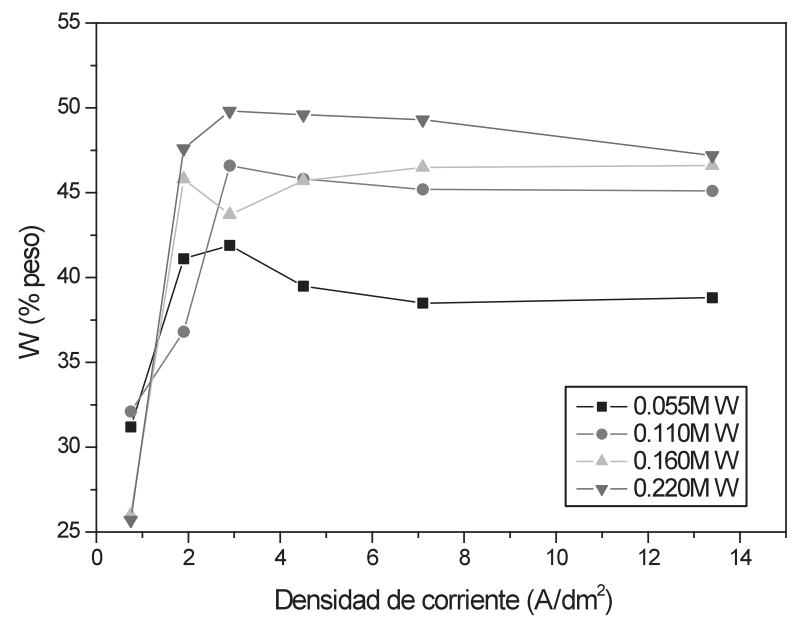

Figura 4. Variación del porcentaje de wolframio (\% en peso) en la aleación de NiW en función de la concentración de wolframio en el electrolito, a una intensidad de $2 \mathrm{~A}$ totales. Condiciones de trabajo: 0,10 $\mathrm{M}$ de níquel, 0,055 a 0,22 $\mathrm{M}$ de wolframio, 0,36 $\mathrm{M}$ de citrato amónico y 0,3 M de compuesto estabilizante. Temperatura $65^{\circ} \mathrm{C}$, $\mathrm{pH} 7$.

Figure 4. Variation of wolfram contained in the NiW alloy (\% in weight), as a function of the concentration of wolfram, at a total intensity of 2 A. Working conditions: $0.10 \mathrm{M}$ of nickel, 0.055 to $0.22 \mathrm{M}$ of wolfram, $0.36 \mathrm{M}$ of ammonium citrate y $0.3 \mathrm{M}$ of estabilizer. Temperature $65^{\circ} \mathrm{C}$, pH 7.

\subsubsection{Influencia de la temperatura}

Partiendo de un baño con 0,10 M de sulfato de níquel, 0,16 M de wolframato sódico, condiciones en las que se obtiene un buen balance espesor-composición, 0,36 M de citrato amónico y 0,3 M de compuesto estabilizante, se ha variado la temperatura del electrolito entre 25 y $75^{\circ} \mathrm{C}$. En las figuras 5 y 6 se representa la variación del espesor del depósito y de la composición de la aleación con la temperatura a diferentes densidades de corriente.

En la figura 5, se puede observar que, a densidades de corriente por debajo de $6 \mathrm{~A} / \mathrm{dm}^{2}$ no hay influencia de la temperatura sobre el espesor del recubrimiento. Sin embargo a densidades de corrientes mayores obtenemos mayores espesores a temperaturas superiores a $65^{\circ} \mathrm{C}$.

En la figura 6 se puede apreciar que, en todos los casos, el porcentaje de wolframio se mantiene constante a partir de una densidad de corriente de 3 $\mathrm{A} / \mathrm{dm}^{2}$ y este porcentaje es mayor cuanto mayor es 


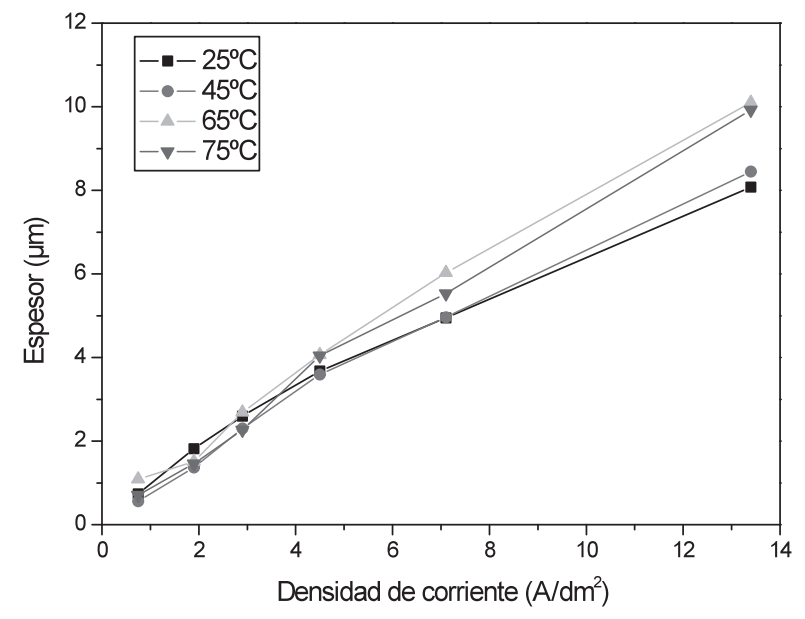

Figura 5. Variación del espesor del depósito de NiW en función de la temperatura, a una intensidad de 2 A totales. Condiciones de trabajo: $0,10 \mathrm{M}$ de níquel, $0,16 \mathrm{M}$ de wolframio, 0,36 $\mathrm{M}$ de citrato amónico y $0,3 \mathrm{M}$ de compuesto estabilizante. Temperatura entre 25 y $75^{\circ} \mathrm{C}, \mathrm{pH} 7$.

Figure 5. Variation of the thickness of the NiW deposit as a function of temperature, at a total intensity of $2 \mathrm{~A}$. Working conditions: $0.10 \mathrm{M}$ of nickel, $0.16 \mathrm{M}$ of wolfram, $0.36 \mathrm{M}$ of ammonium citrate y $0.3 \mathrm{M}$ of estabilizer. Temperature from 25 to $65^{\circ} \mathrm{C}, \mathrm{pH} 7$.

la temperatura de trabajo, no habiendo grandes diferencias a partir de $65^{\circ} \mathrm{C}$.

\subsubsection{Influencia del $p H$}

Partiendo de un baño con 0,10 M de sulfato de níquel, 0,16 M de wolframato sódico, 0,36 M de citrato amónico y 0,3 $\mathrm{M}$ de compuesto estabilizante, se ha variado el $\mathrm{pH}$ del electrolito entre 5 y 9 . En las figuras 7 y 8 se representa la variación del espesor del depósito y de la composición de la aleación con el $\mathrm{pH}$ a diferentes densidades de corriente.

En la figura 7 se puede ver que el espesor del depósito aumenta al aumentar el $\mathrm{pH}$ del electrolito, obteniendo los mayores rendimientos a $\mathrm{pH}$ 9. Sin embargo, se ha observado que, en estas condiciones, el $\mathrm{pH}$ del electrolito es inestable y varía rápidamente con el tiempo de electrolisis.

El efecto del $\mathrm{pH}$ sobre el porcentaje de la aleación se representa en la figura 8 . Puede verse que a pH 5,0 el porcentaje de wolframio no es estable y varía con la densidad de corriente. $\mathrm{A} \mathrm{pH} \mathrm{9,0} \mathrm{el} \mathrm{porcen-}$ taje de wolframio permanece constante pero a valores de alrededor de $30 \%$. Por tanto, el mejor balance

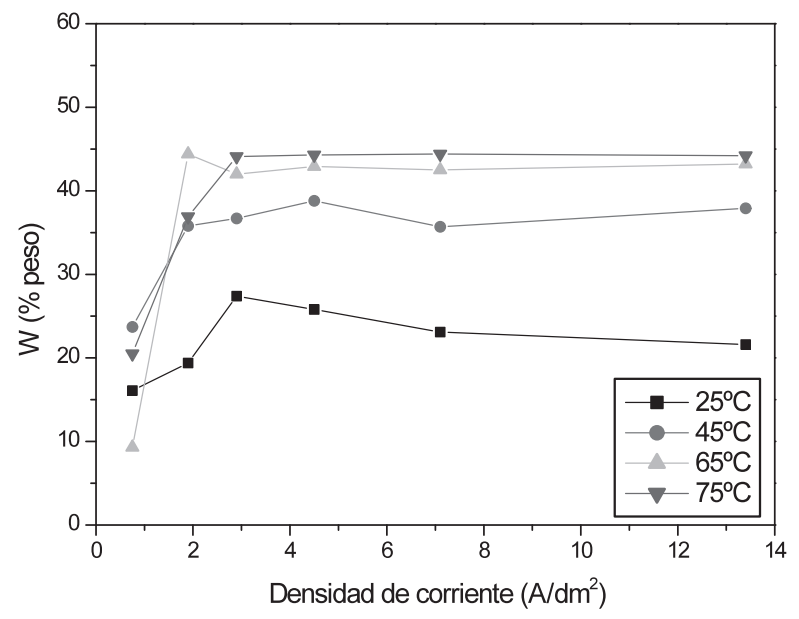

Figura 6. Variación del porcentaje de wolframio en la aleación de NiW en función de la temperatura en el electrolito, a una intensidad de 2 A totales. Condiciones de trabajo: $0,10 \mathrm{M}$ de níquel, $0,16 \mathrm{M}$ de wolframio, $0,36 \mathrm{M}$ de citrato amónico y $0,3 \mathrm{M}$ de compuesto estabilizante. Temperatura entre 25 y $75^{\circ} \mathrm{C}, \mathrm{pH} 7$.

Figure 6. Variation of the percentage of wolfram (\% in weight) of the NiW alloy as a function of the temperature of the electrolyte, at a total intensity of $2 \mathrm{~A}$. Working conditions: $0.10 \mathrm{M}$ of nickel, $0.16 \mathrm{M}$ of wolfram, $0.36 \mathrm{M}$ of ammonium citrate y $0.3 \mathrm{M}$ of estabilizer. Temperature from 25 to $65^{\circ} \mathrm{C}, \mathrm{pH} 7$.

entre espesor y composición se ha obtenido trabajando a $\mathrm{pH} 7,0$.

\subsection{Evaluación de las propiedades de los depósitos de NiW}

En función de los resultados obtenidos con el estudio en célula Hull, se ha definido la composición del electrolito y las condiciones de trabajo, tal y como se describe en el apartado 2.2. A partir de los depósitos obtenidos en estas condiciones se procedió a la evaluación de las propiedades del recubrimiento de NiW.

\subsubsection{Morfología}

Mediante microscopía electrónica de barrido, se estudió el recubrimiento tanto en superficie (Fig. 9) como en sección (Fig. 10). El análisis en sección de la probeta se llevó a cabo mediante criofractura. En la figura 9 se muestra el aspecto superficial de la 


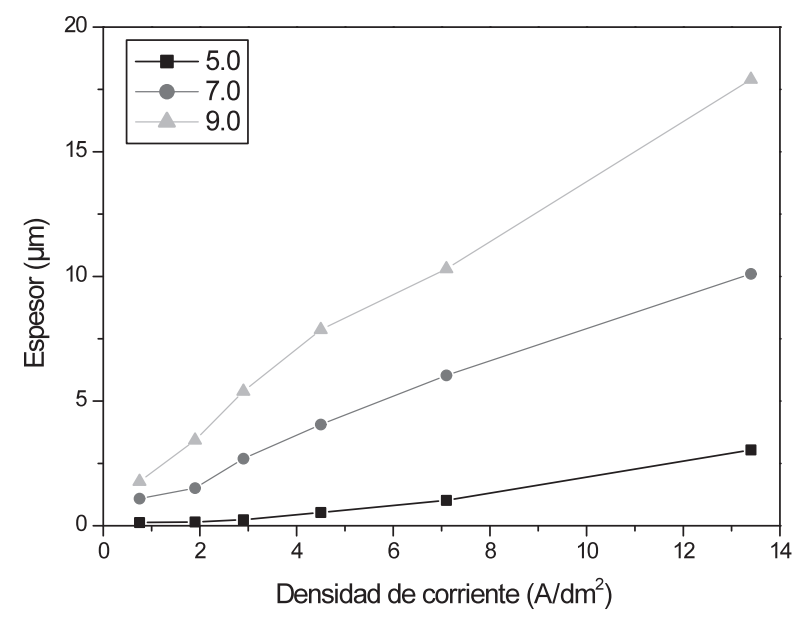

Figura 7. Variación del espesor del depósito de $\mathrm{NiW}$ en función del $\mathrm{pH}$, a una intensidad de 2 A totales. Condiciones de trabajo: $010 \mathrm{M}$ de níquel, $016 \mathrm{M}$ de wolframio, $0,36 \mathrm{M}$ de citrato amónico y $0,3 \mathrm{M}$ de compuesto estabilizante. Temperatura $65^{\circ} \mathrm{C}, \mathrm{pH}$ entre 5 y 9 .

Figure 7. Variation of the thickness of the NiW deposit as a function of $\mathrm{pH}$, at a total intensity of 2 A. Working conditions: $0.10 \mathrm{M}$ of nickel, 0.16 $\mathrm{M}$ of wolfram, $0.36 \mathrm{M}$ of ammonium citrate y 0.3 M of estabilizer. Temperature $75^{\circ} \mathrm{C}, \mathrm{pH}$ from 5 to 9 .

aleación, donde se puede observar que la superficie es globular, poco rugosa y presenta pequeños poros que podrían atribuirse a la inclusión de hidrógeno generado durante el proceso de electrodeposición.

En cuanto a la criofractura realizada (Fig. 10), se puede observar que el recubrimiento es compacto, homogéneo en toda su sección y con buena adherencia con el material base.

\subsubsection{Resistencia a la abrasión}

La evaluación de la resistencia a la abrasión se realizó mediante el ensayo Taber. La pérdida de peso media a los 10.000 ciclos para las probetas obtenidas a partir de las condiciones descritas en el apartado 2.2., fue de 50-60 mg, lo que indica un índice Taber de 56. Este valor es superior al de un recubrimiento de cromo duro, cuyo índice Taber se sitúa en torno a 4.

\subsubsection{Resistencia a la corrosión}

La evaluación de la resistencia a la corrosión en cámara de niebla salina se ha realizado sobre 4 probetas

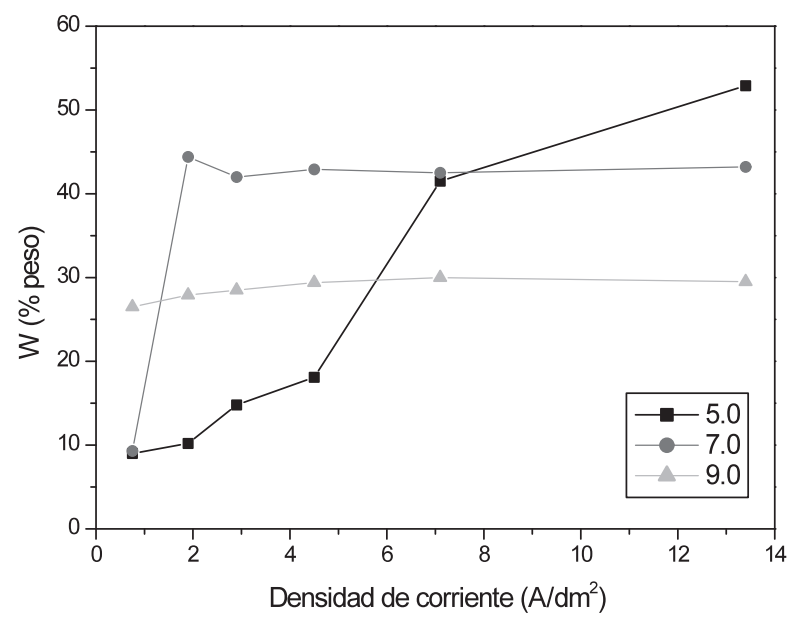

Figura 8. Variación del porcentaje de wolframio (\% en peso) en la aleación de NiW en función del $\mathrm{pH}$ en el electrolito, a una intensidad de $2 \mathrm{~A}$ totales. Condiciones de trabajo: 0,10 M de níquel, 0,16 $\mathrm{M}$ de wolframio, $0,36 \mathrm{M}$ de citrato amónico y 0,3 $\mathrm{M}$ de compuesto estabilizante. Temperatura $65^{\circ} \mathrm{C}, \mathrm{pH}$ entre 5 y 9 .

Figure 8. Variation of the percentage of wolfram (\% in weight) of the NiW alloy as a function of the $\mathrm{pH}$ of the electrolyte, at a total intensity of 2 A. Working conditions: $0.10 \mathrm{M}$ of nickel, 0.16 $\mathrm{M}$ of wolfram, $0.36 \mathrm{M}$ of ammonium citrate y 0.3 $M$ of estabilizer. Temperature $75^{\circ} \mathrm{C}, \mathrm{pH}$ from 5 to 9 .

recubiertas con $\mathrm{NiW}$, bajo las condiciones descritas en el apartado 2.2.

Los resultados mostraron, a las 200 h, la aparición de pocos puntos aislados de corrosión. A las $400 \mathrm{~h}$ de ensayo, la corrosión roja no había progresado en otros puntos de las probetas (Fig. 11). El ensayo finalizó a las 700 h de ensayo, sin apreciarse un avance generalizado de la corrosión.

\subsubsection{Dureza}

Para evaluar la dureza se empleó un microdurómetro Vickers, con carga de 0,98 N. Los resultados del ensayo mostraron que el recubrimiento de $\mathrm{NiW}$ presentaba una dureza de $684 \mathrm{Hv}$. Este valor es inferior a los actuales recubrimientos de cromo duro (1.000 $\mathrm{Hv}$ ); sin embargo, se han conseguido durezas de hasta $1.200 \mathrm{Hv}$ con la aplicación de un tratamiento térmico posterior a $400^{\circ} \mathrm{C}$, durante $1 \mathrm{~h}$ en horno de vacío. 


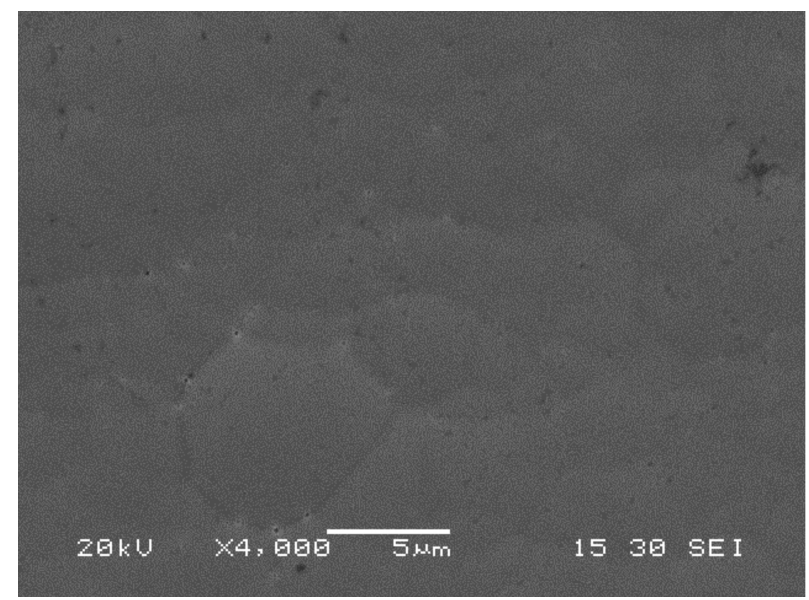

Figura 9. Micrografía SEM en superficie de un recubrimiento de NiW, obtenido a partir de un electrolito que contiene $0,10 \mathrm{M}$ de níquel, $0,16 \mathrm{M}$ de wolframio, 0,36 $\mathrm{M}$ de citrato amónico y 0,3 $\mathrm{M}$ de compuesto estabilizante. Temperatura 65 ${ }^{\circ} \mathrm{C}, \mathrm{pH}$ 7. Densidad de corriente $5 \mathrm{~A} / \mathrm{dm}^{2}$, tiempo de deposición 90 min. Espesor de depósito 25-30 $\mu \mathrm{m}$.

Figure 9. SEM Microscopy of the surface of the NiW alloy, obtained from an electrolyte containing $0.10 \mathrm{M}$ of nickel, $0.16 \mathrm{M}$ of wolfram, $0.36 \mathrm{M}$ of ammonium citrate y $0.3 \mathrm{M}$ of stabilizer. Temperature $65^{\circ} \mathrm{C}, \mathrm{pH}$ 7. Current density $5 \mathrm{~A} / \mathrm{dm}^{2}$, deposition time 90 min. Coating thickness 25-30 $\mu \mathrm{m}$.

\section{CONCLUSIONES}

La célula Hull ha permitido un seguimiento macroscópico de la influencia de la concentración de los metales que componen el baño, para las distintas densidades de corriente, sobre el espesor del depósito y la composición de la aleación. Las gráficas espesor-densidad de corriente demuestran que es el níquel el que mayor influencia tiene sobre el espesor del depósito, siendo recomendable trabajar a concentraciones superiores a $0,068 \mathrm{M}$ de níquel para obtener un rendimiento aceptable. Sin embargo, es la concentración de wolframio la que influye sobre el porcentaje de la aleación, siendo necesario emplear concentraciones superiores a $0,055 \mathrm{M}$ de wolframio para obtener porcentajes de $45 \%$ wolframio en la aleación. Asimismo, se ha comprobado que a bajas densidades de corriente, por debajo de $3-4 \mathrm{~A} / \mathrm{dm}^{2}$, el porcentaje de la aleación no se mantiene constante. Trabajando a valores superiores a $4 \mathrm{~A} / \mathrm{dm}^{2}$ es posible obtener una aleación con un porcentaje constante

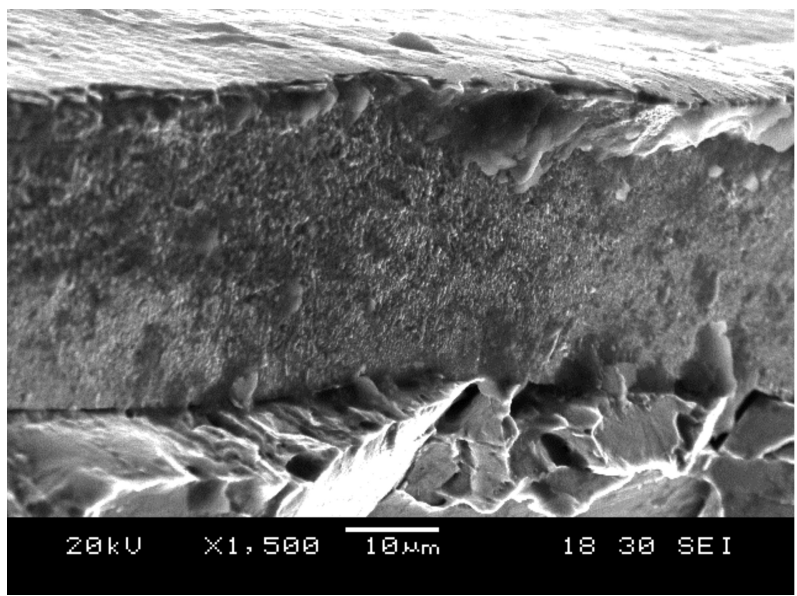

Figura 10. Fractografía en sección del recubrimiento de NiW, obtenido a partir de un electrolito que contiene $0,10 \mathrm{M}$ de níquel, $0,16 \mathrm{M}$ de wolframio, 0,36 $\mathrm{M}$ de citrato amónico y $0,3 \mathrm{M}$ de compuesto estabilizante. Temperatura $65^{\circ} \mathrm{C}, \mathrm{pH}$ 7. Densidad de corriente $5 \mathrm{~A} / \mathrm{dm}^{2}$, tiempo de deposición $90 \mathrm{~min}$. Espesor de depósito 25-30 $\mu \mathrm{m}$.

Figure 10. Fracture study of a cross section of a NiW deposit, obtained from an electrolyte containing $0.10 \mathrm{M}$ of nickel, $0.16 \mathrm{M}$ of wolfram, $0.36 \mathrm{M}$ of ammonium citrate y $0.3 \mathrm{M}$ of stabilizer. Temperature $65^{\circ} \mathrm{C}, \mathrm{pH}$ 7. Current density 5 $\mathrm{A} / \mathrm{dm}^{2}$, deposition time $90 \mathrm{~min}$. Coating thickness 25-30 $\mu \mathrm{m}$.

de wolframio (\% en peso) de alrededor del $45 \%$. En cuanto a la temperatura y el $\mathrm{pH}$, queda demostrado que es necesario trabajar a temperaturas de alrededor de $65^{\circ} \mathrm{C}$ y pH 7,0 para obtener un alto porcentaje de wolframio en la aleación y constante en un amplio rango de densidades de corriente.

La evaluación de las propiedades demuestra que los depósitos obtenidos son compactos, con una morfología globular y con buena adherencia con el material base. Poseen una alta resistencia a la corrosión, superando las 600 h en niebla salina sin corrosión generalizada, una buena resistencia a la abrasión y una dureza que llega a valores de $1.200 \mathrm{Hv}$ tras la aplicación de un tratamiento térmico posterior.

\section{Agradecimientos}

Agradecemos a la empresa Ikan-Kronitek todo su apoyo prestado en la realización de este trabajo. 


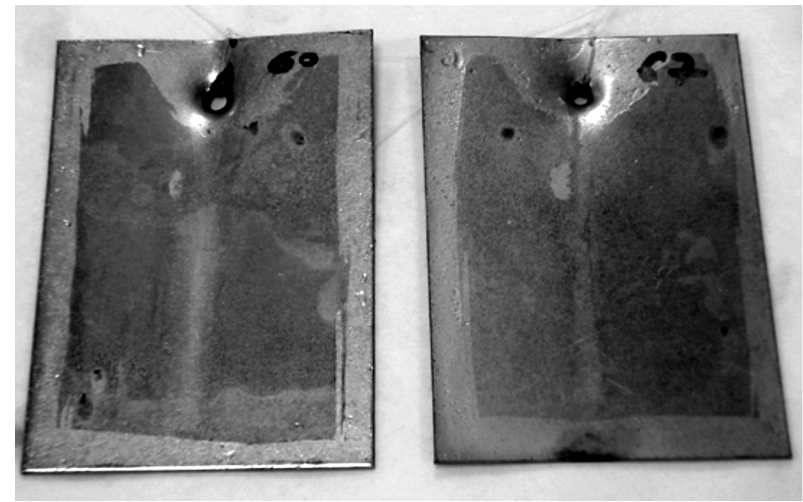

Figura 11. Aspecto del recubrimiento de NiW tras $400 \mathrm{~h}$ de ensayo en niebla salina. Recubrimiento obtenido a partir de un electrolito que contiene $0,10 \mathrm{M}$ de níquel, $0,16 \mathrm{M}$ de wolframio, 0,36 $\mathrm{M}$ de citrato amónico y $0,3 \mathrm{M}$ de compuesto estabilizante. Temperatura $65^{\circ} \mathrm{C}, \mathrm{pH}$ 7. Densidad de corriente $5 \mathrm{~A} / \mathrm{dm}^{2}$, tiempo de deposición $90 \mathrm{~min}$. Espesor de depósito 25-30 $\mu \mathrm{m}$.

Figure 11. Condition of the NiW coating after 400 $h$ in a salt spray chamber. Coating obtained from an electrolyte containing $0.10 \mathrm{M}$ of nickel, 0.16 $M$ of wolfram, $0.36 \mathrm{M}$ of ammonium citrate y 0.3 M of stabilizer. Temperature $65^{\circ} \mathrm{C}, \mathrm{pH}$ 7. Current density $5 \mathrm{~A} / \mathrm{dm}^{2}$, deposition time $90 \mathrm{~min}$. Coating thickness 25-30 $\mu \mathrm{m}$.

\section{REFERENCIAS}

[1] E. George, Electrodeposited Alloys as an Alternative for Hexavalent Chromium, Atotech, South Carolina, EE. UU., 2003, pp. 1-8.

[2] A. Brenner, Electrodeposition of Alloys. Principles and Practice Vol. I, Academic Press, New York, EE. UU., 1963, pp. 75-80.

[3] O. Younes y E. Gieladi, J. Electrochem. Soc. 149 (2002) C100-C111.

[4] L. Zhu, O. Younes, N. Ashkenasy, Y. ShachamDiamand y E. Gileadi, Appl. Surf. Sci. 200 (2002) $1-4$.

[5] M. Obradovic, J. Stevanovic, R. Stevanovic y A. Despic, J.Electroanal. Chem. 491 (2000) 188196.

[6] M.D. Obradovic, R.M. Stevanivic y A.R. Despic, J.Electroanal. Chem. 552 (2003) 185-196.

[7] V. Massuet, La célula Hull para el control de Baños Galvánicos, Ed. Cedel, Barcelona, España, 1969, pp. 11-17.

[8] A.C. West, M. Matlosz y D. Landolt, J.Appl. Electrochem. 22 (1992) 301-303.

[9] R.O.Hull, Am. Electroplat. Soc. 27 (1939) 5257. 